\title{
Strategies for Theory Assessment and Enhancement in Marketing: An Abstract
}

\author{
Matthew Wilson and Jeannette Paschen
}

\begin{abstract}
Developing theoretical contributions in marketing is challenging for any scholar but can be particularly difficult for doctoral students or recent graduates. Many marketing academics have received reviews of articles they submitted to scholarly marketing journals only to find their work rejected or required to undergo revisions because of issues related to theory. Given the often incremental nature of theory contributions, it is difficult to understand what the threshold is for a clear and sufficient theoretical contribution in the marketing discipline. Our paper aims to address this challenge by elucidating what, exactly, a theoretical contribution in marketing is, in addition to offering practical advice to new and established marketing scholars on how to develop it.

In doing so, our work provides two contributions: First, we outline why theoretical contributions are difficult to develop in general and especially in marketing. To further elucidate how to overcome these challenges, we showcase three exemplary papers that have advanced marketing theory and highlight elements of these contributions that make them so valuable. Second, our manuscript provides guidelines for authors regarding theoretical sufficiency and significance of their work that authors can consult prior to submitting their work to an academic journal for peer review.
\end{abstract}

References Available Upon Request

M. Wilson $(\bowtie) \bullet J$. Paschen

Royal Institute of Technology (KTH), Stockholm, Sweden

e-mail: matthew.wilson@indek.kth.se; jeannette.paschen@indek.kth.se 